\title{
Exact results for scattering on ultrashort plane wave backgrounds
}

\author{
A. Ilderton $\odot^{*}$ \\ Centre for Mathematical Sciences, University of Plymouth, Plymouth, PL4 8AA, United Kingdom
}

(Received 6 September 2019; published 20 December 2019)

\begin{abstract}
We give exact results for the emission spectra of both nonlinear Breit-Wheeler pair production and nonlinear Compton scattering in ultraintense, ultrashort duration plane wave backgrounds, modeled as delta-function pulses. This includes closed form expressions for total scattering probabilities. We show explicitly that these probabilities do not exhibit the power-law scaling with intensity associated with the conjectured breakdown of (Furry picture) perturbation theory, instead scaling logarithmically in the highintensity limit.
\end{abstract}

DOI: $10.1103 /$ PhysRevD.100.125018

\section{INTRODUCTION}

The coupling of matter to a background field can be arbitrarily strong, requiring nonperturbative methods in the calculation of scattering amplitudes on the background. Highly symmetric backgrounds such as electromagnetic plane waves [1-3], widely used as a first model of intense laser fields [4-7], may be treated exactly for arbitrarily high field strength, allowing a great deal of analytic progress to be made in the calculation of scattering amplitudes.

However, the evaluation of observables still requires intense numerical integration. While various results have been found which allow more efficient calculation [8-10], it would be desirable to have exact results when confronting questions of a fundamental nature, such as the limits of perturbation theory in the high intensity regime; it has been conjectured that (Furry picture [11]) perturbation theory in strong fields breaks down for sufficiently high field strengths, due to a power-law scaling of higher loop processes [12-16]. If true, this would invalidate all current perturbative or semiperturbative approaches to QED in strong backgrounds $[17,18]$ - it is thus crucial to understand precisely when the conjecture does and does not hold.

Here we have two objectives. The first is to provide some exact results for two scattering processes on ultrashort plane wave backgrounds, namely nonlinear Compton scattering $[4,5,7]$ and nonlinear Breit-Wheeler, or stimulated, pair production $[4,5,19]$. By exact we mean that, although we work to first order in the fine structure constant $\alpha$, our results are exact in the coupling to the background

\footnotetext{
*anton.ilderton@plymouth.ac.uk
}

Published by the American Physical Society under the terms of the Creative Commons Attribution 4.0 International license. Further distribution of this work must maintain attribution to the author(s) and the published article's title, journal citation, and DOI. Funded by SCOAP. field, and also of closed form-it is this latter property which is usually unobtainable, but which is key to fulfilling our second objective: this is to obtain the high-intensity scaling of our results in light of the conjectured highintensity breakdown.

The model on which the conjecture is based, and the starting point for many investigations of laser-matter interactions, assumes the laser fields may be treated as constant and homogeneous [4]. We consider here the opposite limit in which the field is of infinitely short duration, being modeled as a sequence of delta function pulses. The reason for focussing on this limit is that it will allow us to provide the kind of exact and closed-form results we need. (See [20] for the use of delta pulses, in combination with constant fields, to Schwinger pair production, where exact results are also obtained.)

This paper is organized as follows. In Sec. I A we review some necessary structures of QED scattering in plane wave backgrounds. In Sec. II we consider stimulated pair production in a delta-function pulse. Both the differential emission spectra and the total pair creation probability are obtained in closed form, with all integrals performed. We show that the high-intensity scaling of the probability is logarithmic, not power law. We then consider the case of two delta function pulses modeling an oscillating field. In Sec. III we turn to nonlinear Compton scattering, and find that the leading order, high intensity, behavior in an oscillating field is doubly logarithmic. We conclude in Sec. IV.

\section{A. General structures}

Consider the scattering of some collection of particles incident on a classical background field, which we here take to be a plane wave. The wave is described by the potential $e A_{\mu}=a_{\mu}(n . x)$ where $n_{\mu}$ is null, i.e. $n^{2}=0$. We work in lightfront coordinates, $x^{ \pm}:=x^{0} \pm x^{3}$ and $x^{\perp}=$ $\left\{x^{1}, x^{2}\right\}$. We can always choose $n \cdot x=x^{+}$. We can also 
choose the potential to have only nonzero "transverse" components $a_{\perp}$, and to obey $a_{\perp}(-\infty)=0$ [21]; the derivative of $a_{\perp}$ then gives the electric field of the wave, while $a_{\perp}$ itself describes the work done on a particle entering the wave from $n \cdot x=-\infty$ [22-24].

Scattering amplitudes in plane wave backgrounds are calculated in the Furry picture [11], see [7] for a recent introduction. In this approach, the coupling of matter to the classical background is treated as part of the "free theory," meaning that it affects the fermion propagator and the asymptotic wavefunctions (external legs of Feynman diagrams). The latter are known as "Volkov solutions" [1], call them $\psi(x)$; they can be written down without approximation for plane waves of arbitrary strength and profile, and obey the Dirac equation in that background, i.e. $(\not \not \partial-m-\not h) \psi=0$. We illustrate the possible solutions with that describing an outgoing positron which has asymptotic, final momentum $q_{\mu}$, as will be useful below; defining $\delta a_{\mu}(\phi)=a_{\mu}(\phi)-a_{\mu}(\infty)$, this is

$$
\begin{aligned}
\psi_{q}(x)= & {\left[1-\frac{\not h \delta \phi(\phi)}{2 n \cdot q}\right] v_{q} } \\
& \times \exp \left[i q \cdot x-i a(\infty) \cdot x+\frac{i}{2 n \cdot q} \int_{\phi}^{\infty} 2 q \cdot \delta a+\delta a^{2}\right],
\end{aligned}
$$

in which $v_{q}$ is the standard Dirac spinor [25].

In the Furry picture, interactions between quantum particles, i.e. the fermions and dynamically generated/ absorbed photons, are as usual treated in perturbation theory with respect to the fine structure constant $\alpha$. Here we work to lowest order in $\alpha$, which for the processes of interest corresponds to tree level. To illustrate, we use one of the processes on which we focus below, namely stimulated pair production. Consider a photon, momentum $l_{\mu}$ and polarization $\epsilon_{\mu}$, incident on a plane wave. Through interaction with this background, the photon produces an electronpositron pair of momenta $p_{\mu}$ and $q_{\mu}$ respectively. We write $\psi_{p}(x)$ and $\psi_{q}(x)$ for the corresponding Volkov solutions. Then the order- $\alpha$ scattering amplitude takes the form

$$
\begin{aligned}
S_{f i} & =i e \int \mathrm{d}^{4} x \bar{\psi}_{p}(x) \phi e^{-i l . x} \psi_{q}(x) \\
& =\frac{i e}{2}(2 \pi)^{3} \delta_{-, \perp}^{3}(q+p-l) \mathcal{M} .
\end{aligned}
$$

In the second equality, the symmetry properties of plane waves $[2,3]$ lead to the overall conservation of longitudinal $\left(p_{-}:=\left(p_{0}-p_{3}\right) / 2\right)$ and transverse $\quad\left(p_{\perp}:=\left\{p_{1}, p_{2}\right\}\right)$ momenta. The nontrivial part of the scattering amplitude, $\mathcal{M}$, is an integral over lightfront time $\phi \equiv n . x$, of the form [21]

$$
\mathcal{M}=-\int \mathrm{d} \phi e^{i \Phi} \frac{\mathrm{d}}{\mathrm{d} \phi}\left[\frac{\operatorname{Spin}(a)}{i \Phi^{\prime}}\right]
$$

in which $\Phi$ is a real function of $\phi$ with nonvanishing $\phi$ derivative, denoted $\Phi^{\prime}$, and "Spin" is a combination of spin and polarization structures which depends on $\phi$ through the background $a_{\mu}(\phi)$; both derive from the Volkov solutions. The explicit forms of $\Phi$ and Spin will be given below for the cases of interest. ${ }^{1}$

Several kinds of integral arise in the calculation of scattering probabilities in plane waves. Integrals over transverse final state momenta are always Gaussian [8], and integrals over longitudinal momenta (of form n.p) can often be performed to give special functions $[8,29]$. However the $\phi$-integral in $\mathcal{M}$ cannot be performed analytically in general. There are two special cases, constant and monochromatic fields, for which symmetry allows the $\phi$-integral to be performed. However, the resulting change in form then prohibits the final state momentum integrals from being performed. In contrast to this, we will in the examples below be able to perform the $\phi$-integrals, and more, exactly.

\section{PAIR PRODUCTION IN DELTA-FUNCTION PULSES}

We consider the production of an electron-positron pair, momenta $p_{\mu}$ and $q_{\mu}$ respectively, from a photon of momentum $l_{\mu}$ incident on a plane wave pulse. We take the electric field of the pulse to be a single peak as illustrated in Fig. 1. Such fields, called unipolar [30] or impulsive, transfer net energy-momentum to a classical particle traversing them, via a nonzero Fourier zero mode of the electric field [21]. (See [31-36] for various applications in gravity.) The specific case of interest here is a linearly polarized electric field with $E_{2}=a_{2}=0$ and, for $m$ the electron mass,

$$
\begin{aligned}
E_{1}(\phi) & =\frac{m \omega \xi}{2} \operatorname{sech}^{2}(\omega \phi), \\
a_{1}(\phi) & =m \xi(1+\tanh (\omega \phi)) .
\end{aligned}
$$

In the limit $\omega \rightarrow \infty$, see also Fig. $1, E_{1}$ becomes a delta function and the potential becomes a step function,

$$
E_{1}(\phi) \rightarrow m \xi \delta(\phi), \quad a_{1}(\phi) \rightarrow m \xi H(\phi) .
$$

Our chosen parametrization corresponds to holding fixed, in this limit, the product of the peak electric field $E_{0} \sim \omega \xi$, and the effective temporal duration $\sim 1 / \omega$. In other words

\footnotetext{
${ }^{1}$ Two subtleties compared to standard literature calculations are that (i) we use the modified LSZ prescription detailed in $[21,26]$ in order to account for unipolar fields for which the background potential may not vanish asymptotically, and (ii) we have written $\mathcal{M}$ in a regulated form [21,27] by symbolically subtracting (in a manner consistent with gauge invariance [28]) the $a_{\mu} \rightarrow 0$ amplitude, which is trivially vanishing. The reason is simply that this form is convenient for the calculations below.
} 

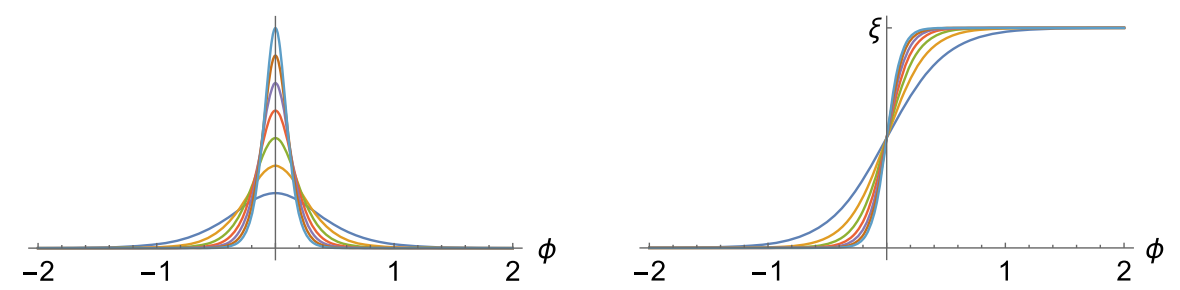

FIG. 1. The electric field (left) and potential (right) for a unipolar field. In the considered limit, the electric field becomes a delta function at the origin, while the potential becomes a Heaviside step function.

we fix the total work done by the field $m \xi=e E_{0} / \omega$; thus $\xi$ matches the usual definition of the dimensionless intensity parameter [4,5]. In order to evaluate the pair production scattering amplitude in this limit, it is helpful to make the boundary terms in (3) explicit. We thus rewrite (3) as

$$
\mathcal{M}=-\left.e^{i \Phi} \frac{\operatorname{Spin}(a)}{i \Phi^{\prime}}\right|_{-X} ^{X}+\int_{-X}^{X} \mathrm{~d} \phi e^{i \Phi} \operatorname{Spin}(a)
$$

in which $X$ is an arbitrary positive constant. In the limit that the electromagnetic field of the pulse becomes a delta function, the Volkov solutions yield the following explicit expressions for $\Phi$ and Spin, with $a_{\mu}=m \xi \delta_{\mu}^{1}$ (for $\delta_{\nu}^{\mu}$ the Kronecker delta) now a constant:

$$
\begin{aligned}
\Phi & = \begin{cases}\frac{l . q}{n \cdot l-n \cdot q} \phi, & \phi>0 \\
\frac{l . \bar{\pi}}{n \cdot l-n \cdot q} \phi, & \phi<0\end{cases} \\
\operatorname{Spin}(a) & = \begin{cases}\bar{u}_{p} \notin v_{q} & \phi>0 \\
\bar{u}_{p}\left(1-\frac{d \varkappa}{2 n \cdot p}\right) \notin\left(1+\frac{n \alpha}{2 n \cdot q}\right) v_{q} & \phi<0,\end{cases}
\end{aligned}
$$

in which $u_{p}$ is the standard Dirac spinor [25] and the phase $\Phi$ is expressed in terms of

$$
\bar{\pi}^{\mu}:=q^{\mu}-a^{\mu}+n^{\mu} \frac{2 q \cdot a-a^{2}}{2 n \cdot q} .
$$

The interpretation of $\bar{\pi}_{\mu}$ is that it is the initial momentum of a classical positron which has traversed the delta function pulse and has final momentum $q_{\mu}$. With these results, the $\mathrm{d} \phi$-integral in (6) can be performed by splitting the integration into the ranges $\phi>0$ and $\phi<0$. The boundary terms at $\pm X$ are then canceled, and we lose (as we should) all dependence on the arbitrary $X$. What remains is

$$
\mathcal{M} \rightarrow \frac{\operatorname{Spin}_{<}}{i \Phi_{<}^{\prime}}-\frac{\operatorname{Spin}_{>}}{i \Phi_{>}^{\prime}}
$$

in which the subscripts $\gtrless$ refer to evaluation at $\phi>0$ and $\phi<0$, where both Spin and $\Phi$ are constants. This compact and exact result is easily understood; it means that we only pick up contributions from across the delta function at the origin, where the arguments of $\Phi$ and Spin jump. This is typical of situations with delta functions, and is physically sensible as it means we pick up contributions only from where the field strength is nonzero.

From here we mod-square, and average (sum) over initial (final) polarizations and spins. The resulting expression for the pair production probability is, in terms of the positron's final momentum components $q_{\perp}$ and $u:=n . q / n . l$,

$$
\begin{aligned}
\mathbb{P}= & \frac{\alpha m^{2}}{4 \pi^{2}} \int \mathrm{d}^{2} q_{\perp} \int_{0}^{1} \frac{\mathrm{d} u}{u}(1-u) \\
& \times\left[\frac{1}{(l \cdot \bar{\pi})^{2}}+\frac{1}{(l \cdot q)^{2}}-\frac{2}{l \cdot \bar{\pi} l \cdot q}\left(1+\xi^{2} h(u)\right)\right],
\end{aligned}
$$

in which $h(u)=1 / 2-1 / 4 u(1-u)$. The result is, as may be expected, very similar to that obtained for scattering of a particle off an instantaneous kick, as used to exemplify infrared divergences of QED [25]. (There is no infrared divergence here.) Figure 2, left panel, shows an example of the differential emission probability

$$
\frac{u}{1-u} \frac{\mathrm{d}^{3} \mathbb{P}}{\mathrm{d}^{2} q_{\perp} \mathrm{d} u} .
$$

In the figure we take $u=1 / 2$, the "symmetric point" which corresponds to the positron and electron each carrying half of the initial photon's lightfront momentum n.l. Taking $l_{\perp}=0$, describing a head-on collision between the photon and plane wave, we observe two peaks in the positron spectrum, at momenta $q_{\perp}=0$ and $q_{\perp}=a_{\perp}$. A "semiclassical" explanation of this result is as follows. Assume that all particles are created at zero transverse momentum. Then positrons created in the rise of the ultrashort pulse (of which we are taking a limit) are accelerated by practically the whole field and so pick up the full possible transverse momentum $a_{\perp}$ from it. Hence the spectral peak at $q_{\perp}=a_{\perp}$. Positrons created as the field falls, on the other hand, see little of it and therefore pick up little momentum after creation. This gives the spectral peak at $q_{\perp}=0$.

\section{A. Total probability}

In contrast to other cases, we can here perform all the final state integrals in (10) and so obtain a closed form 

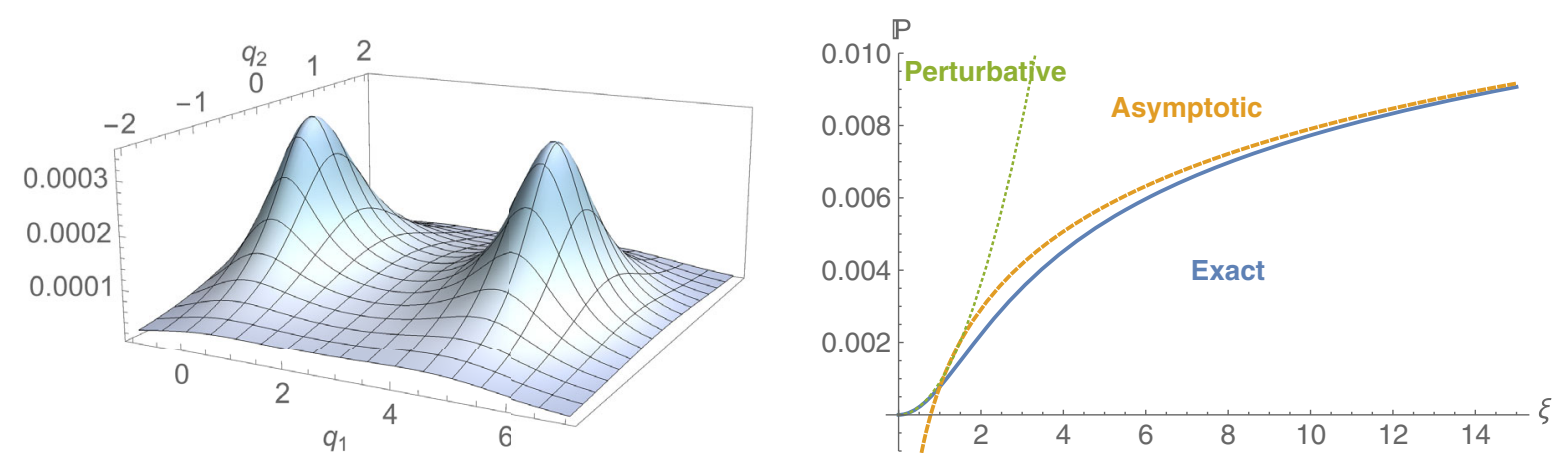

FIG. 2. Left: an example of the differential emission probability (11) in the delta pulse, as a function of the created positron's final transverse momenta $q_{1}$ and $q_{2}$ (all in units of the electron mass $m$ ), at the symmetric point $u=1 / 2$, which corresponds to the produced particles each carrying half of the initial photon's lightfront momentum. (We take $l_{\perp}=0$.) The intensity parameter is $\xi=5$. The two peaks in the spectrum at $q_{\perp}=0$ and $q_{\perp}=a_{\perp}=\{m \xi, 0\}$ correspond to pairs produced at zero transverse momentum in the instantaneous rise and instantaneous fall of the field. Right: the total pair creation probability, along with its small $\xi$ and large $\xi$ approximations.

expression for the total pair production probability. Consider the three terms in large square brackets in (10). In the first term, make a change of variables from $q_{\perp}$ to $v_{\perp}$ defined by $q_{\perp}=m v_{\perp}+u l_{\perp}+a_{\perp}$. Then that term becomes

$$
\frac{\alpha}{\pi^{2}} \int \mathrm{d}^{2} v_{\perp} \int_{0}^{1} \mathrm{~d} u \frac{u(1-u)}{\left(1+\left|v_{\perp}\right|^{2}\right)^{2}}=\frac{\alpha}{6 \pi} .
$$

This is independent of $\xi$. A similar change of variable shows that the second term in (10) gives the same contribution. For the final term in (10), we make the same change of variable as above. The $u$-integral is then trivial, the angular integration in the $v_{\perp}$ plane may be performed using tangent half-angle substitution, see [37] Sec. III.613, and the $\left|v_{\perp}\right|$ integral can then be performed exactly (see below for details). The total probability is

$$
\begin{aligned}
\mathbb{P}= & \frac{\alpha}{3 \pi}+\frac{\alpha}{3 \pi} \frac{\xi^{2}-1}{\xi \sqrt{\xi^{2}+4}} \\
& \times \log \left[1+\frac{\xi^{4}}{2}+2 \xi^{2}+\xi \sqrt{\xi^{2}+4}+\frac{1}{2} \xi^{3} \sqrt{\xi^{2}+4}\right] .
\end{aligned}
$$

This exact result is plotted in Fig. 2, right panel. We observe that the probability is completely independent of the initial photon energy. This reflects the fact that, because the delta pulse contains all (arbitrarily high) frequency modes which can contribute their energy to the process, a photon of any energy is capable of producing pairs in the background. While our result is exact for a delta-function pulse, it is also a first approximation to the probability in ultrashort pulses; we conclude that in such pulses the total probability of pair production will depend only weakly on initial photon energy. Note that our result does not imply a nonzero probability in the absence of the initial photon, which would be unphysical since a single plane wave cannot spontaneously produce pairs [38]; we have assumed at almost all stages of the calculation that $l_{\mu} \neq 0$ (i.e. when dividing by $l . q$, when introducing $u=n . q / n . l$, and so on). Expanding (13) for small $\xi$ shows that the probability behaves as

$$
\mathbb{P} \simeq \frac{7}{18} \frac{\alpha}{\pi} \xi^{2}, \quad \xi \ll 1 .
$$

We now turn to the question of how the probability (13) behaves at high intensity, or large $\xi$, in light of the conjecture discussed in the introduction. Processes in constant crossed fields (the zero frequency limit of plane waves) can scale at high intensity with powers of $\xi^{2 / 3}[12-16]$. If one invokes the "locally constant field approximation" (LCFA) [4] then the power-law scaling generalizes to more general fields $[39,40]$. This has lead to the conjecture that for sufficiently high $\xi$ the Furry expansion as used here breaks down. Due to the importance of both understanding the conjecture, and due to the many shortcomings of the LCFA [39-44] it would though be desirable to have exact analytic results from which asymptotic scalings could be obtained unambiguously. ${ }^{2}$ As we have a closed form result, (13), we may simply expand it for large $\xi$, finding

$$
\mathbb{P} \simeq \frac{\alpha}{3 \pi}+\frac{4}{3} \frac{\alpha}{\pi} \log \xi, \quad \xi \gg 1
$$

Thus the probability for stimulated pair production scales logarithmically, asymptotically; there is no power-law scaling as for the same process in constant crossed fields (or as

\footnotetext{
${ }^{2}$ The breakdown is normally discussed in the context of loops. One-loop processes, which already exhibit the $\xi^{2 / 3}$ scaling in the LCFA, [12-16] are by the optical theorem related to the tree-level process discussed above and below; studying these processes is therefore already enough to identify differences compared to constant fields or the LCFA.
} 

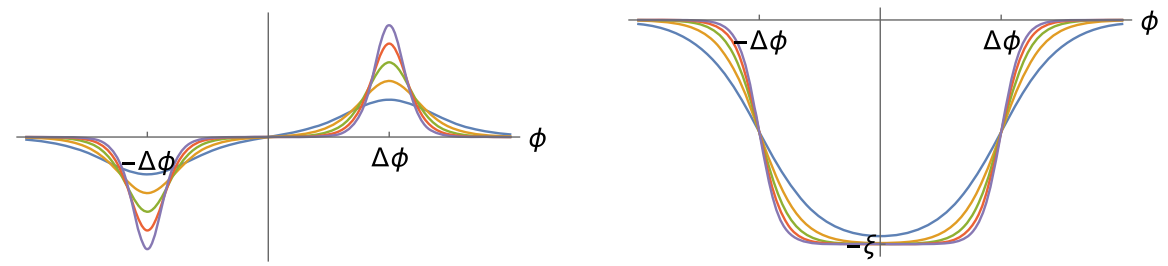

FIG. 3. Left: the electric field which, in the considered limit, becomes a sequence of two delta functions of opposite sign. Right: in the same limit, the potential becomes a box function with support on $|\phi|<\Delta \phi$.

predicted by the LCFA, which is clearly not valid for short pulses). It is interesting that this exact result shows a logarithmic behavior more typical of QED. The small and large $\xi$ approximations are plotted in Fig. 2. Despite the complexity of the argument of the logarithm in (13), the exact result is very well approximated by the large $\xi$ expansion (15) for $\xi>10$.

\section{B. Quantum interference effects in pair production}

We turn now to a background composed of two delta pulses, as in (5), of opposite sign and separated by a (lightfront) time delay of $2 \Delta \phi$, see Fig. 3. In this case the field has no dc component, meaning that, classically (and ignoring radiation and backreaction), a particle traversing the whole field would pick up zero net energy-momentum. This is usually assumed when modeling laser pulses, which are naturally oscillating fields. In this case, the pair production amplitude is found to be

$$
\begin{aligned}
\mathcal{M} & \rightarrow\left(\int_{-\Delta \phi}^{\Delta \phi} \mathrm{d} \phi e^{i \Phi}\right)\left[\operatorname{Spin}(a)-\frac{l \cdot \bar{\pi}}{l \cdot p} \operatorname{Spin}(0)\right] \\
& =2 \sin \left[\frac{\Delta \phi l \cdot \bar{\pi}}{n \cdot l(1-u)}\right]\left(\frac{\operatorname{Spin}_{<}}{\Phi_{<}^{\prime}}-\frac{\operatorname{Spin}_{>}}{i \Phi_{>}^{\prime}}\right),
\end{aligned}
$$

in which all the variables, $\bar{\pi}, \Phi_{\gtrless}$ and so on, are exactly as for the single delta pulse above. It follows that the emission spectrum and probability are given simply by inserting the factor $4 \sin ^{2}$ under the integrals of (10):

$$
\begin{aligned}
\mathbb{P}= & \frac{\alpha m^{2}}{4 \pi^{2}} \int \mathrm{d}^{2} q_{\perp} \int_{0}^{1} \frac{\mathrm{d} u}{u}(1-u) 4 \sin ^{2}\left[\frac{\Delta \phi l \cdot \bar{\pi}}{n \cdot l(1-u)}\right] \\
& \times\left(\frac{1}{(l \cdot \bar{\pi})^{2}}+\frac{1}{(l \cdot q)^{2}}-\frac{2}{l \cdot q l \cdot \bar{\pi}}\left[1+\xi^{2} h(u)\right]\right) .
\end{aligned}
$$

The difference compared to the case of a single delta pulse is the $4 \sin ^{2}$ interference factor, which represents a coherent enhancement of the rate through quantum interference [45,46]. The pair spectrum (11) is illustrated in Fig. 4. Note that the spectral peaks remain at the same positions $q_{\perp}=0$ and $q_{\perp}=a_{\perp}$ as before. Using the above semiclassical picture and referring to Fig. 3, positrons created in the rise of the first peak are accelerated and decelerated by the whole field, picking up $-a_{\perp}$ in transverse momentum at the first delta, and then $+a_{\perp}$ at the second, thus ending with zero transverse momentum. Positrons created in the fall of the second peak receive no momentum from the field. These two creation events interfere and source the spectral peak at $q_{\perp}=0$. Positrons created in the fall of the first delta, or the rise of the second, pick up only $+a_{\perp}$ in transverse momentum from the second delta. These two events interfere and source the second spectral peak at $q_{\perp}=a_{\perp}$. The degree of interference in the spectrum is controlled by both the separation of the delta pulses and the energy of the initial photon; these appear in the argument of the $\sin ^{2}$ factors in the dimensionless combination $\theta:=m^{2} \Delta \phi / n . l$. For small $\theta$ the pair production probability is suppressed, because (see Fig. 3) the field vanishes in this limit.

For $\theta$ large, the differential spectrum exhibits extremely rapid oscillations due to interference. In the same limit, these effects drop out of the total probability as follows; make the same change of variables from $q_{\perp}$ to $v_{\perp}$ as above equation (12), then the interference factor becomes

$$
4 \sin ^{2}\left[\frac{\theta}{2} \frac{1+v^{2}}{u(1-u)}\right]=2-2 \cos \left[\theta \frac{1+v^{2}}{u(1-u)}\right] .
$$

The argument of the cosine is at least $4 \theta$ and (as will be made clear below) the remaining $v$-integral in $\mathbb{P}$ is dominated by contributions from $v \sim \xi$; hence if $\xi$ is large

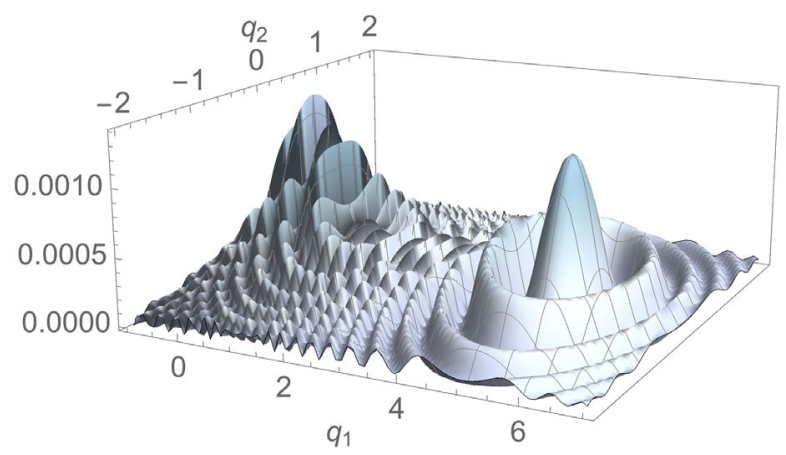

FIG. 4. Differential emission probability (11) as a function of transverse momenta (all in units of electron mass $m$ ) at $u=1 / 2$, with $\xi=5, \theta=1$, in two delta pulses, the first negative and the second positive. The spectrum is identically equal to that in a single positive delta peak, multiplied by the $4 \sin ^{2}$ interference term. 
and $\theta$ not too small, then to a first approximation the cosine factor is very rapidly oscillating, and integrating over it will give zero contribution to the total probability. What remains is the leading 2 in (18); hence, in the large $\xi$ limit, interference effects drop out of the integrated probability, which becomes approximately equal to twice that in a single delta (15). We will see in the next section that there are processes for which interference effects "persist," leading to a different high intensity scaling in the total probability. Interference effects in pair production will be discussed in more detail elsewhere [47].

\section{NONLINEAR COMPTON SCATTERING}

We now consider nonlinear Compton scattering (NLC), that is the emission of a photon, momentum $l_{\mu}$, from an electron of momentum $p_{\mu}$ incident on the classical plane wave background [4,5,7]. We will express the probability in terms of the produced photon's transverse momentum $l_{\perp}$ and its longitudinal momentum fraction $s:=n . l / n . p$. We begin again with a single delta pulse as in Fig. 1. This field is unipolar, which means the NLC emission probability will show the usual logarithmic divergence of QED in the infrared [21]. Due to the symmetry properties of the plane wave, it is convenient to regulate this divergence using a small cutoff $\varepsilon_{\mathrm{IR}}$ in the lightfront momentum fraction $s$ of the emitted photon, rather than in its frequency. With this, a similar calculation to that for pair production leads to the emission probability

$$
\begin{aligned}
\mathbb{P}_{\mathrm{NLC}}= & -\frac{\alpha m^{2}}{4 \pi^{2}} \int \mathrm{d}^{2} l_{\perp} \int_{\varepsilon_{\mathrm{IR}}}^{1} \frac{\mathrm{d} s}{s}(1-s) \\
& \times\left[\frac{1}{(l \cdot \pi)^{2}}+\frac{1}{(l \cdot p)^{2}}-\frac{2}{l \cdot \pi l \cdot p}\left(1+\xi^{2} g(s)\right)\right],
\end{aligned}
$$

in which, for $a_{\mu}=m \xi \delta_{\mu}^{1}$ again,

$\pi^{\mu}=p^{\mu}+a^{\mu}+\frac{-2 a \cdot p-a^{2}}{2 n \cdot p} n^{\mu}, \quad g(s):=\frac{1}{2}+\frac{1}{4} \frac{s^{2}}{1-s}$.

The calculation of the integrals in (19) differs from those for (13) only in the $s$-integration, which is trivial. We therefore quote the exact final result, dropping only terms which vanish as $\varepsilon_{\mathrm{IR}} \rightarrow 0$ :

$$
\begin{aligned}
\mathbb{P}_{\mathrm{NLC}}= & \frac{2 \alpha}{\pi}\left(1+\log \varepsilon_{\mathrm{IR}}\right) \\
& -\frac{2 \alpha}{\pi} \frac{\log [\text { pair }]}{\xi \sqrt{\xi^{2}+4}}\left(1+\frac{3}{8} \xi^{2}+\left(1+\frac{1}{2} \xi^{2}\right) \log \varepsilon_{\mathrm{IR}}\right),
\end{aligned}
$$

in which "pair" indicates precisely the same argument as for pair production (13). The essential difference is clearly only in the dependence on the small $s$ cutoff. The leading behavior for $\xi \gg 1$ is again logarithmic,

$\mathbb{P}_{\mathrm{NLC}} \sim \frac{2 \alpha}{\pi}\left(1+\log \varepsilon_{\mathrm{IR}}\right)-\frac{4 \alpha}{\pi}\left(\frac{3}{4}+\log \varepsilon_{\mathrm{IR}}\right) \log \xi$.

We note that, by the optical theorem and replacing $\varepsilon_{\mathrm{IR}}$ with a detector resolution $\varepsilon_{\min }$, the scaling (22) also applies to the forward scattering probability $\mathbb{P}_{\text {foward }}$ at one-loop, since to that order $\mathbb{P}_{\text {foward }}=1-\mathbb{P}_{\mathrm{NLC}}$.

\section{A. Total emission probability in alternating sign pulses}

As for pair production, we also consider two alternating sign pulses as in Fig. 3. In this case there is no infra-red divergence. A direct calculation shows that the total probability is again given by inserting a $4 \sin ^{2}$ interference factor into the single-pulse result (19):

$$
\begin{aligned}
\mathbb{P}= & -\frac{\alpha m^{2}}{4 \pi^{2}} \int \mathrm{d}^{2} l_{\perp} \int_{0}^{1} \frac{\mathrm{d} s}{s}(1-s) 4 \sin ^{2}\left[\frac{1}{b} \frac{l . \pi}{m^{2}(1-s)}\right] \\
& \times\left(\frac{1}{(l . \pi)^{2}}+\frac{1}{(l \cdot p)^{2}}-\frac{2}{l \cdot \pi l \cdot p}\left[1+\xi^{2} g(s)\right]\right),
\end{aligned}
$$

in which $1 / b=\Delta \phi m^{2} / n . p$ is the analog of the interference-energy parameter in pair production. ( $\pi^{\mu}$ as defined above may here be interpreted as the Lorentz force momentum of the incoming electron, momentum $p_{\mu}$, between the first and second deltas.) There are clear similarities with pair production, so we focus on the differences, one of which is the high-intensity scaling of the probability. The key observation is the following. We cannot, as we did for pair production, write $4 \sin ^{2} x \rightarrow$ $2-2 \cos 2 x$ and drop the second term as rapidly oscillating. The physical reason is that doing so would introduce a divergence at $s=0$, because the replacement would, in effect, reduce the probability to that in a single delta pulse.

To see what is happening, we change variables in (23), defining $v_{\perp}:=l_{\perp} / s-\pi_{\perp}$, and we express the integral in terms of the modulus $v$ and polar angle $\vartheta$ of $v_{\perp}$. We consider the final term in the large round brackets of (23) which (we will see) is the dominant term. In terms of our new variables the integral to be performed is

$$
\begin{aligned}
\mathbb{P}= & \frac{8 \alpha}{\pi^{2}} \int_{0}^{\infty} \frac{\mathrm{d} v v}{1+v^{2}} \int_{0}^{1} \frac{\mathrm{d} s}{s}(1-s)\left(1+\xi^{2} g(s)\right) \sin ^{2}\left[\frac{s\left(1+v^{2}\right)}{2 b(1-s)}\right] \\
& \times \int_{0}^{2 \pi} \mathrm{d} \vartheta \frac{1}{1+v^{2}+\xi^{2}-2 \xi v \cos \vartheta}+\ldots
\end{aligned}
$$

Consider the $\sin ^{2}$ factor; independent of the value of $b$ and $v^{2}$, the factor $s /(1-s)$ can still be arbitrarily small, and hence there is a portion of the integration range (small $s$ ) over which $\sin ^{2}$ oscillates arbitrarily slowly, in contrast to pair production. This demonstrates why we cannot simplify 
the $4 \sin ^{2}$ factor to an overall 2 as we did for pair production. Now, we again use tangent half-angle substitution to perform the $\vartheta$-integral,

$$
\begin{aligned}
\mathbb{P}= & \frac{16 \alpha}{\pi} \int_{0}^{\infty} \frac{\mathrm{d} v}{1+v^{2}} \frac{v}{\sqrt{\xi^{4}-2 \xi^{2}\left(v^{2}-1\right)+\left(1+v^{2}\right)^{2}}} \\
& \times \int_{0}^{1} \frac{\mathrm{d} s}{s}(1-s)\left(1+\xi^{2} g(s)\right) \sin ^{2}\left[\frac{s\left(1+v^{2}\right)}{2 b(1-s)}\right]+\ldots
\end{aligned}
$$

The $s$-integral can be performed exactly, but is an unenlightening combination of Sine- and Cosine-integrals. However, this function is well approximated by its asymptotic expansion for large argument, i.e. when $x \gtrsim 1$,

$$
\begin{aligned}
& \int_{0}^{1} \frac{\mathrm{d} s}{s}(1-s)\left(1+\xi^{2} g(s)\right) \sin ^{2}\left[\frac{s x}{2(1-s)}\right] \\
& \simeq \frac{1}{4} \xi^{2}\left(\log (x)+\gamma_{E}-\frac{3}{4}\right)+\frac{1}{2}\left(\log (x)+\gamma_{E}-1\right) .
\end{aligned}
$$

It is sufficient to use this approximation in establishing the leading order behavior for $\xi \gg 1$, for the following reasons. The relevant argument for us is $x=\left(1+v^{2}\right) / b$ which is at least equal to $1 / b$. Inspection of (25) shows that the remaining $v$-integral is strongly peaked around $v \simeq 1$ and $v \simeq \xi$. The height of the former peak is independent of $\xi$, whereas the latter increases with $\xi$ and hence gives the leading order large $\xi$ contribution; this behavior is the same when using (26). Hence we substitute (26) into (25). Define $\xi_{*}=\sqrt{\xi^{2}+4}$ [which has already appeared in (13)] and change variables from $v$ to $t$ defined by $1+v^{2}=\xi \xi_{*} / t$; with this, the integrals to be performed are

$$
\begin{aligned}
\mathbb{P} & \sim \frac{8 \alpha}{\pi \xi \xi_{*}} \int_{0}^{\xi \xi_{*}} \frac{\mathrm{d} t}{\sqrt{1+t^{2}-2 t \xi / \xi_{*}}}\left(k_{1}-k_{2} \log t\right) \\
& =: \frac{8 \alpha}{\pi \xi \xi_{*}}\left(k_{1} I_{1}-k_{2} I_{2}\right),
\end{aligned}
$$

which defines the integrals $I_{1}$ and $I_{2}$, and where the constants $k_{j}$ are determined by (26) to be, writing down only the dominant contributions,

$k_{2} \simeq \frac{1}{4} \xi^{2}, \quad k_{1} \simeq \frac{1}{4} \xi^{2} \log \frac{\xi \xi_{*}}{b} \sim \frac{1}{2} \xi^{2} \log \xi$ for $\xi \gg 1$.

The integral $I_{1}$ may be calculated exactly (and is also that needed for pair production in a single delta pulse):

$I_{1}=\log \left[1+\xi \xi_{*}+\frac{\xi^{3} \xi_{*}}{2}+\frac{\xi^{2} \xi_{*}^{2}}{2}\right] \sim 4 \log \xi$ for $\xi \gg 1$.

We have not found a closed form expression for the remaining integral $I_{2}$. However, we can find the leading order behavior for large $\xi$, as follows. As $\xi \rightarrow \infty$, the ratio $\xi / \xi_{*}$ under the square root approaches unity at leading order, and the integrand jumps at $t=1$ but remains integrable. In this limit the only dependence on $\xi$ is in the upper integral limit. Hence we can obtain the leading order contribution by approximating

$$
I_{2} \rightarrow \int_{0}^{\xi^{2}} \mathrm{~d} t \frac{\log t}{|1-t|}=-\frac{\pi^{2}}{6}-\operatorname{Li}_{2}\left(1-\xi^{2}\right) \sim 2 \log ^{2} \xi,
$$

in which $\mathrm{Li}$ is the polylogarithm. We are finally in a position to evaluate (27). Combining (28)-(30) we obtain

$$
\begin{aligned}
\mathbb{P} & \sim \frac{8 \alpha}{\pi \xi^{2}}\left(\frac{1}{2} \xi^{2} \log \xi \cdot 4 \log \xi-\frac{1}{4} \xi^{2} \cdot 2 \log ^{2} \xi\right) \\
& =\frac{12 \alpha}{\pi} \log ^{2} \xi+\ldots
\end{aligned}
$$

There are two further terms to calculate in (23). The first term in large round brackets there is independent of $\xi$. The calculation of the second term proceeds similarly to that above; the $\vartheta$-integral is very similar, the $s$-integral is the same, and may again be approximated by its large argument expansion. The resulting $v$-integral can also be performed, and the resulting contribution goes like $\log \left(\xi^{2} / b\right)$, which (like all the energy dependence) is subleading compared to (31).

Thus, (31) gives the asymptotic scaling of the full probability, and is doubly logarithmic. The difference in scaling compared to pair production (double rather than single logarithm) is due to the different infrared behaviors of the two processes; in effect, it seems that it is not possible to completely suppress interference effects in NLC, and this is what changes the asymptotic scaling. The obtained scaling is also notable in comparison to other results in the literature. For constant crossed fields, the asymptotic scaling is $\mathbb{P} \sim\left(\xi^{2} / b\right)^{1 / 3}$, and the same is implied to hold for pulses by invoking the LCFA. We have now seen explicitly, for both nonlinear Compton scattering and pair production, as well as the one-loop effects connected to them via the optical theorem, that there is no such scaling in the ultrashort pulses considered here.

\section{CONCLUSIONS}

We have considered scattering processes in strong plane wave backgrounds. It is well known that the amplitudes for such processes contain highly oscillatory, cumbersome phase integrals which require numerical integration. By using delta functions to model the limit of ultrashort pulses, we have been able to perform these integrals exactly, and so evaluate without approximation the emission spectra of nonlinear Breit Wheeler pair production and nonlinear Compton scattering. We have also explained the main features of the emission spectrum in terms of an intuitive physical picture. We remark that the extension of our calculations to sequences of three or more delta pulses is straightforward, yet contains a surprisingly rich structure, see [47]. 
In some cases it is also possible to perform all final state momentum integrals exactly, and thus obtain exact, closed form expressions for total scattering probabilities as a function of initial parameters, in particular the field intensity. We have seen that the power-law scaling with intensity inferred from constant crossed field results is completely absent, replaced with a logarithmic scaling. While this may not be entirely surprising, given the obvious differences between constant fields and delta functions, it illustrates unambiguously that the conjectured high-intensity scaling does not hold for arbitrary pulses. Thus our results complement those of $[39,40]$ in showing that the conjectured scaling, and the implied breakdown of perturbation theory, are not universal.

We note further that for both pair production and nonlinear Compton, it was the study of the low (lightfront) energy part of the emission spectra, and the damping or persistence of interference effects there, which allowed us to establish the high-intensity scalings; this is particularly interesting because it is in this part of the spectrum that the locally constant field approximation fails [41]. The potential implications for the NR conjecture will be discussed elsewhere.
While constant fields are unphysical in the infrared, our delta pulses should be expected to be unphysical in the UV. (This is however not an issue for the high intensity scaling in which we are primarily interested here $[39,40]$.) One goal for future work is therefore the identification of other backgrounds, neither infinitely long nor short, which also allow all integrals to be performed analytically [9]. Finding such examples may be challenging, given the complexity evident in e.g. (13) even for the case of delta-function pulses with little internal structure. However, such examples would provide us with improved insight into physics in strong fields, allow us to better check numerical methods and approximations [41-43], and could also provide particular data to help analyse the double copy [48-52] of gauge theory on background plane waves $[36,53,54]$. It would also be very interesting to see how much progress can be made with challenging higher-order (many vertex) processes in delta-pulse backgrounds.

\section{ACKNOWLEDGMENTS}

A. I. is supported by The Leverhulme Trust, project grant RPG-2019-148.
[1] D. M. Volkov, Z. Phys. 94, 250 (1935).

[2] V. G. Bagrov and D. M. Gitman, Exact Solutions of Relativistic Wave Equations (Springer, New York, 1990).

[3] T. Heinzl and A. Ilderton, Phys. Rev. Lett. 118, 113202 (2017).

[4] V. I. Ritus, J. Russ. Laser Res. 6, 497 (1985).

[5] A. Di Piazza, C. Muller, K. Z. Hatsagortsyan, and C. H. Keitel, Rev. Mod. Phys. 84, 1177 (2012).

[6] B. King and T. Heinzl, High Power Laser Sci. Eng. 4, e5 (2016).

[7] D. Seipt, in Proceedings, Quantum Field Theory at the Limits: from Strong Fields to Heavy Quarks (HQ 2016): Dubna, Russia, 2016 (2017), pp. 24-43 [arXiv:1701.03692].

[8] V. Dinu, Phys. Rev. A 87, 052101 (2013).

[9] D. Seipt, V. Kharin, S. Rykovanov, A. Surzhykov, and S. Fritzsche, J. Plasma Phys. 82, 655820203 (2016).

[10] V. Dinu and G. Torgrimsson, Phys. Rev. D 99, 096018 (2019).

[11] W. H. Furry, Phys. Rev. 81, 115 (1951).

[12] V. I. Ritus, Sov. Phys. JETP 30, 1181 (1970).

[13] D. Morozov and N. Narozhny, Sov. Phys. JETP 45, 23 (1977).

[14] N. B. Narozhnyi, Phys. Rev. D 20, 1313 (1979).

[15] N. B. Narozhnyi, Phys. Rev. D 21, 1176 (1980).

[16] D. Morozov and N. Narozhny, Sov. Phys. JETP 53, 1103 (1981).

[17] A. M. Fedotov, J. Phys. Conf. Ser. 826, 012027 (2017).

[18] NP-QED Workshop, SLAC, https://conf.slac.stanford.edu/ npqed-2019/ (2019).
[19] H. R. Reiss, J. Math. Phys. (N.Y.) 3, 59 (1962).

[20] A. M. Fedotov and A. A. Mironov, Phys. Rev. A 88, 062110 (2013).

[21] V. Dinu, T. Heinzl, and A. Ilderton, Phys. Rev. D 86, 085037 (2012).

[22] S. J. Brodsky, H.-C. Pauli, and S. S. Pinsky, Phys. Rep. 301, 299 (1998).

[23] T. Heinzl, Lect. Notes Phys. 572, 55 (2001).

[24] B. L. G. Bakker et al., Nucl. Phys. B, Proc. Suppl. 251-252, 165 (2014).

[25] M. E. Peskin and D. V. Schroeder, An Introduction to Quantum Field Theory (Addison-Wesley, Reading, MA, 1995).

[26] T. W. B. Kibble, Phys. Rev. 138, B740 (1965).

[27] M. Boca and V. Florescu, Phys. Rev. A 80, 053403 (2009).

[28] A. Ilderton, Phys. Rev. Lett. 106, 020404 (2011).

[29] V. Dinu, T. Heinzl, A. Ilderton, M. Marklund, and G. Torgrimsson, Phys. Rev. D 89, 125003 (2014).

[30] V. V. Kozlov, N. N. Rosanov, C. De Angelis, and S. Wabnitz, Phys. Rev. A 84, 023818 (2011).

[31] P. C. Aichelburg and R. U. Sexl, Gen. Relativ. Gravit. 2, 303 (1971).

[32] R. Penrose, in General Relativity: Papers in Honour of J.L. Synge, edited by L. O'Raifeartaigh (Springer, New York, 1972), pp. 101-115.

[33] T. Dray and G. 't Hooft, Nucl. Phys. B253, 173 (1985).

[34] C. Klimcik, Phys. Lett. B 208, 373 (1988). 
[35] V. Ferrari, P. Pendenza, and G. Veneziano, Gen. Relativ. Gravit. 20, 1185 (1988).

[36] T. Adamo, E. Casali, L. Mason, and S. Nekovar, Classical Quantum Gravity 35, 015004 (2018).

[37] I. Gradshteyn and I. Ryzhik, Table of Integrals, Series, and Products (Academic Press, San Diego, CA, 2007).

[38] J. S. Schwinger, Phys. Rev. 82, 664 (1951).

[39] T. Podszus and A. Di Piazza, Phys. Rev. D 99, 076004 (2019).

[40] A. Ilderton, Phys. Rev. D 99, 085002 (2019).

[41] C. N. Harvey, A. Ilderton, and B. King, Phys. Rev. A 91, 013822 (2015).

[42] A. Di Piazza, M. Tamburini, S. Meuren, and C. H. Keitel, Phys. Rev. A 98, 012134 (2018).

[43] A. Ilderton, B. King, and D. Seipt, Phys. Rev. A 99, 042121 (2019).

[44] B. King, B. M. Dillon, K. A. Beyer, and G. Gregori, arXiv:1905.05201.

[45] F. Hebenstreit, R. Alkofer, G. V. Dunne, and H. Gies, Phys. Rev. Lett. 102, 150404 (2009).
[46] E. Akkermans and G. V. Dunne, Phys. Rev. Lett. 108, 030401 (2012).

[47] A. Ilderton, arXiv:1910.03012.

[48] Z. Bern, J. J. M. Carrasco, and H. Johansson, Phys. Rev. D 78, 085011 (2008).

[49] Z. Bern, J. J. M. Carrasco, and H. Johansson, Phys. Rev. Lett. 105, 061602 (2010).

[50] Z. Bern, T. Dennen, Y.-T. Huang, and M. Kiermaier, Phys. Rev. D 82, 065003 (2010).

[51] J. J. M. Carrasco, in Proceedings, Journeys through the Precision Frontier: Amplitudes for Colliders (TASI 2014): Boulder, Colorado, 2014 (WSP, 2015), pp. 477-557 [arXiv: 1506.00974].

[52] Z. Bern, J. J. Carrasco, M. Chiodaroli, H. Johansson, and R. Roiban, arXiv:1909.01358.

[53] T. Adamo, E. Casali, L. Mason, and S. Nekovar, J. High Energy Phys. 02 (2019) 198.

[54] T. Adamo and A. Ilderton, J. High Energy Phys. 06 (2019) 015. 